\title{
Pencil-core Granuloma Forming 62 Years After Initial Injury
}

\author{
Blake R. Zelickson, MD, PhD; Leonard H. Goldberg, MD; Wesley Wu, MD; Marc Rubenzik, MD, PhD; \\ Kiran Motaparthi, MD
}

\section{PRACTICE POINTS}

- Pencil-core granulomas can arise even decades after the lead is embedded in the skin.

- It is important to biopsy to confirm the diagnosis, as pencil-core granulomas can very closely mimic melanomas.

To the Editor:

Trauma from a pencil tip can sometimes result in a fragment of lead being left embedded within the skin. Pencil lead is composed of $66 \%$ graphite carbon, $26 \%$ aluminum silicate, and $8 \%$ paraffin. ${ }^{1,2}$ While the toxicity of these individual elements is low, paraffin can cause nonallergic foreign-body reactions, aluminum silicate can induce epithelioid granulomatous reactions, and graphite has been reported to cause chronic granulomatous reactions in the lungs of those who work with graphite. ${ }^{2}$ Penetrating trauma with a pencil can result in the formation of a cutaneous granulomatous reaction that can sometimes occur years to decades after the initial injury.,4 Several cases of pencil-core granulomas have been published, with lag times between the initial trauma and lesion growth as long as 58 years. $^{1-10}$ The pencil-core granuloma may simulate malignant melanoma, as it presents clinically as a growing, darkly pigmented lesion, thus prompting biopsy. We present a case of a pencil-core granuloma that began to grow 62 years after the initial trauma.

A 72-year-old woman was referred to our clinic for evaluation of a dark nodule on the forehead. The lesion had been present since the age of 10 years, reportedly from an accidental stabbing with a pencil. The lesion had been flat, stable, and asymptomatic since the trauma occurred; however, the patient reported that approximately 9 months prior to presentation, it had started growing and became painful. Physical examination revealed a $1.0-\mathrm{cm}$, round, bluish-black nodule on the right superior forehead (Figure 1A). No satellite lesions or local lymphadenopathy were noted on general examination.

An elliptical excision of the lesion with 1-cm margins revealed a bluish-black mass extending through the dermis, through the frontalis muscle, and into the periosteum and frontal bone (Figure 1B). A No. 15 blade was then used to remove the remaining pigment from the outer table of the frontal bone. Histopathologic findings demonstrated a sarcoidal granulomatous dermatitis associated with abundant, nonpolarizable, black, granular pigment consistent with carbon tattoo. This foreign material was readily identifiable in large extracellular deposits and also within histiocytes, including numerous multinucleated giant cells (Figure 2). Immunostaining for MART-1 and SOX-10 antigens failed to demonstrate a melanocytic proliferation. These findings were consistent with a sarcoidal foreign-body granulomatous reaction to carbon tattoo following traumatic graphite implantation.

Granulomatous reactions to carbon tattoo may be sarcoidal (foreign-body granulomatous dermatitis), palisading, or rarely tuberculoid (caseating). Sarcoidal granulomatous tattoo reactions may occur in patients with sarcoidosis due to koebnerization, and histology alone is not discriminatory; however, in our patient, the absence of underlying sarcoidosis or clinical or histologic findings of sarcoidosis outside of the site of the pencil-core granuloma excluded that possibility. ${ }^{11}$ Pencil-core granulomas are characterized by a delayed foreign-body reaction to retained fragments of lead often years following a

Drs. Zelickson, Goldberg, Wu, and Rubenzik are from DermSurgery Associates, Houston, Texas. Dr. Motaparthi is from the Department of Dermatology, University of Florida College of Medicine, Gainesville.

The authors report no conflict of interest.

Correspondence: Leonard H. Goldberg, MD, DermSurgery Associates, 7515 S Main St, Ste 240, Houston, TX 77030 (goldb1@dermsurgery.org). doi:10.12788/cutis.0459 
FIGURE 1. A, A 1.0-cm, round, bluish-black nodule on the right superior forehead. B, Intraoperative view of pigment extending into the underlying frontal bone.

FIGURE 2. A, Low-power view demonstrated a granulomatous dermatitis with abundant pigment. Numerous foreign body-type giant cells and fibrosis were associated with the pigment $(\mathrm{H} \& \mathrm{E}$, original magnification $\times 40$ ).

$\mathrm{B}$, Carbon tattoo and foreignbody reaction extended to the periosteum and bone $(\mathrm{H} \& \mathrm{E}$, original magnification $\times 100$ ).
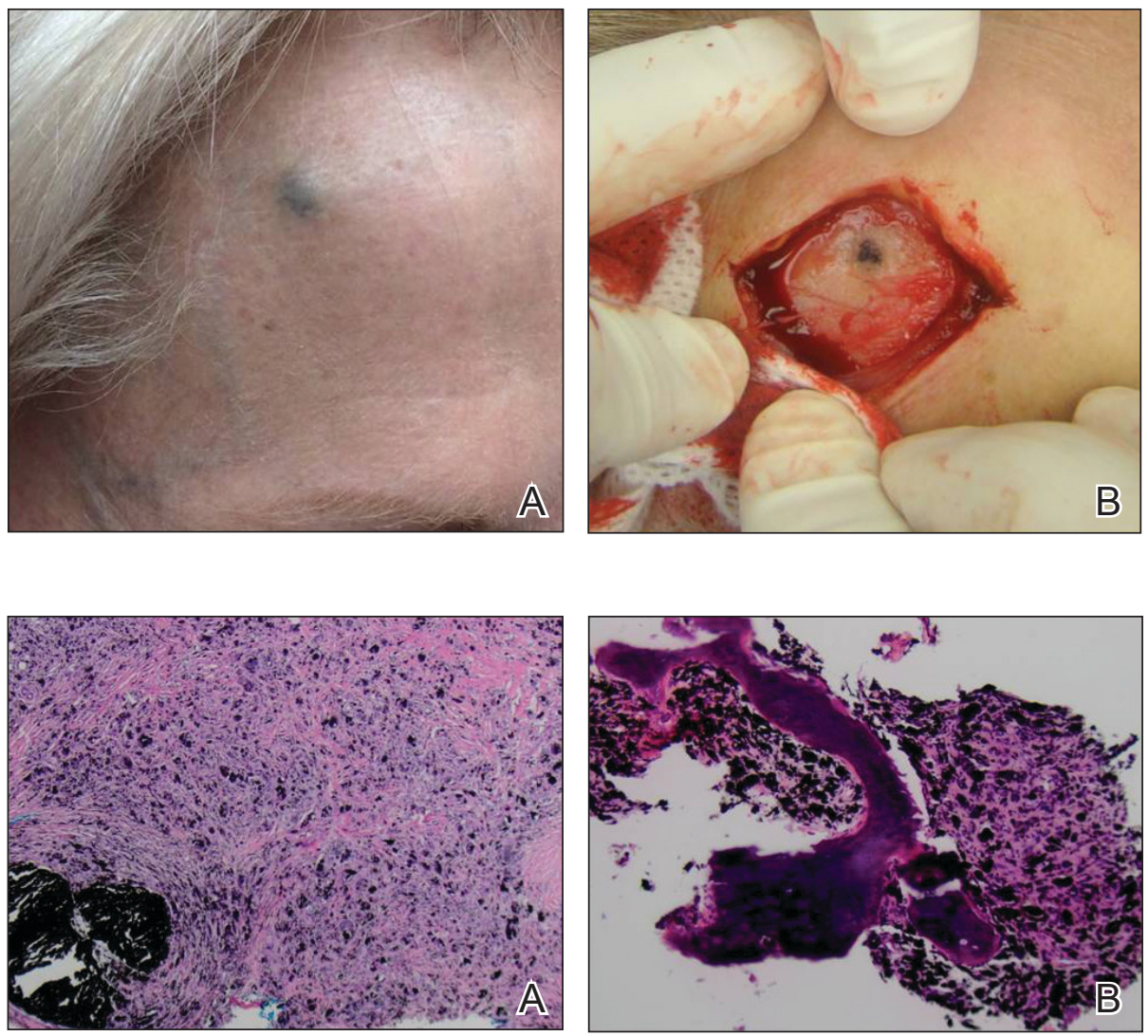

penetrating trauma with a pencil. Previous reports have described various lag times from injury to lesion growth of up to 58 years..$^{1-10}$ Our patient claimed to have noticed the lesion growing and becoming painful only after a 62 -year lag time following the initial trauma. To our knowledge, this is the longest lag time between the initial pencil injury and induction of the foreign-body reaction reported in the literature. Clinically, the lesion appeared and behaved very similar to a melanoma, prompting further treatment and evaluation.

It has been suggested that the lag period between the initial trauma and the rapid growth of the lesion may correspond to the amount of time required for the breakdown of the pencil lead to a critical size followed by the dispersal of those particles within the interstitium, where they can induce a granulomatous reaction. ${ }^{1,2,9}$ One case described a patient who reported that the growth and clinical change of the pencil-core granuloma only started when the patient accidentally hit the area where the trauma had occurred 31 years prior. ${ }^{1}$ This additional trauma may have caused further mechanical breakdown of the lead to set off the tissue reaction. In our case, the patient did not recall any additional trauma to the head prior to the onset of growth of the nodule on the forehead.

Our case indicates that carbon tattoo may be a possible sequela of a penetrating injury from a pencil with retained pencil lead fragments; however, many of these carbon tattoos may remain stable throughout the remainder of the patient's life. Carbon tattoo alone does not necessitate surgical treatment, but when an evolving lesion has a clinical differential diagnosis that includes a melanocytic neoplasia, biopsy or complete removal for histopathologic evaluation is warranted.

\section{REFERENCES}

1. Gormley RH, Kovach SJ III, Zhang PJ. Role for trauma in inducing pencil "lead" granuloma in the skin. J Am Acad Dermatol. 2010;62:1074-1075.

2. Terasawa N, Kishimoto S, Kibe $Y$, et al. Graphite foreign body granuloma. Br J Dermatol. 1999;141:774-776.

3. Fukunaga Y, Hashimoto I, Nakanishi H, et al. Pencil-core granuloma of the face: report of two rare cases. J Plast Reconstr Aesthet Surg. 2011;64:1235-1237.

4. Aswani VH, Kim SL. Fifty-three years after a pencil puncture wound. Case Rep Dermatol. 2015;7:303-305.

5. Taylor B, Frumkin A, Pitha JV. Delayed reaction to "lead" pencil simulating melanoma. Cutis. 1988;42:199-201.

6. Granick MS, Erickson ER, Solomon MP. Pencil-core granuloma. Plast Reconstr Surg. 1992;89:136-138.

7. Andreano J. Stump the experts. foreign body granuloma. J Dermatol Surg Oncol. 1992;18:277, 343.

8. Yoshitatsu S, Takagi T. A case of giant pencil-core granuloma. J Dermatol. 2000;27:329-332.

9. Hatano $Y$, Asada $Y$, Komada S, et al. A case of pencil core granuloma with an unusual temporal profile. Dermatology. 2000;201:151-153.

10. Seitz IA, Silva BA, Schechter LS. Unusual sequela from a pencil stab wound reveals a retained graphite foreign body. Pediatr Emerg Care. 2014;30:568-570.

11. Motaparthi K. Tattoo ink. In: Cockerell CJ, Hall BJ, eds. Nonneoplastic Dermatopathology. 2nd ed. Amirsys; 2016: 270. 